\title{
A novel in-vehicle real-time brake-monitoring system
}

\author{
D E Fumi ${ }^{1 *}$ and I A Sultan ${ }^{2}$

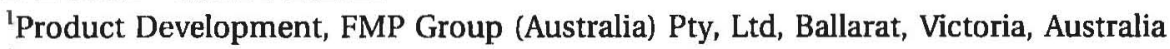 \\ ${ }^{2}$ School of Science and Engineering, University of Ballarat, Ballarat, Victoria, Australia
}

The manuscript was received on 26 August 2008 and was accepted after revision for publication on 27 February 2009.

DOI: 10.1243/09544070JAUTO996

\begin{abstract}
The design of vehicle brake systems involves optimizing a large number of competing parameters. In order to find an appropriate balance of properties, it is important to have a good picture of the range of operating conditions that the system will operate under. Obtaining such information for a large range of driving conditions requires recording data over a long period of time from a number of vehicles under various driving conditions. In this paper, a proposed data acquisition system has been designed, constructed, and implemented to measure and to store in real time the performance-related parameters of brake systems.

The system proposed here to monitor brake performance employs a small embedded personal computer together with a number of peripheral cards as its basis. As a part of the validation process, recordings have been made of some long-term test sequences during a brake development programme. The results, which would be considered representative of normal confident driving, are given and discussed at the end of the paper. These results are used to monitor brake performance and do offer valuable guidance for future brake design projects.
\end{abstract}

Keywords: brake-monitoring system, vehicle brakes, driving conditions, brake performance

\section{INTRODUCTION}

Despite the emergence of many novel safety features, the brake system is still the most important way of avoiding an accident or incident. It has a simple purpose, i.e. to convert the vehicle's kinetic energy into heat and to dissipate the heat in an efficient manner, appropriate to the operating envelope of the vehicle. A challenge to the design process of brake systems is manifested by the fact that driving conditions which the vehicle will experience through its life are not known. This applies to both the input conditions (e.g. pedal forces, speeds, and other factors) and the dependent parameters such as deceleration and brake pad temperature. As such, Chand et al. [1] suggested that brake system design with respect to friction material is typically test centric and follows an

*Corresponding author: Product Development, FMP Group (Australia) Pty, Ltd, 8 Elizabeth St., Ballarat, Victoria, 3350, Australia.

email: duncan.fumi@bendix.com.au iterative development path. The testing procedures are usually designed in such a way that performance parameters are measured and recorded using datalogging equipment. Currently, much of the datalogging equipment required for brake testing has been much specialized and correspondingly expensive. With a good grasp of the usage conditions a brake performance test can be arranged to obtain the most relevant information in the fastest possible time. This suggests a need for data on the factors that underline drivers' behaviour. The lack of reliable and affordable technology to collect such data inspired the development of the system proposed in this paper. An attempt to map drivers' behaviour was presented in a study by Rienstra and Rietveld [2]. Their paper focused on drivers' perception and resulting actions under different speed conditions. Shiiba and Suda [3] developed a driving simulator based on a multi-body vehicle model. Their paper investigates changes in driver response as parameters of the simulation are altered. Igarashi et al. [4] investigated whether an individual's driving behaviour is unique to them and thus identifiable. 
The frequency spectra of brake and accelerator pedal motion were measured from recordings made of a number of drivers, but the findings were not conclusive. The work of Erdogan $e t a l$. [5] has been useful in showing analysis of data on visual, audible, and behavioural analysis of drivers in order to identify the driver positively. On the other hand, Smith et al. [6] described research into the use of a single camera to determine the attentiveness of a vehicle driver based on the head position (rotational and fore-aft).

Other research work in the area of brake performance analysis has been more focused on the technical aspects of the brake system design. For example, Sakamoto [7] discussed the relationship between brake rotor dimensions and heat dissipation problems from both a physical and an experimental perspective. One outcome of this research is that the convection area is the most important factor that controls the effectiveness of the brakes to dissipate heat. On the other hand, Park and Choi [8] investigated the relationship between the brake noise propensity and the rotor runout (misalignment), first, through experimental testing on a dynamometer and, second, via a theoretical three-degrees-of-freedom model of the braking system. Fieldhouse et al. [9] used machine vision in a creative experimental set-up to isolate the in-plane vibration of brake discs and to animate the relevant mode of vibrations. They, therefore, presented a breakthrough in the area of brake noise understanding and control.

The present paper describes a novel data-logging system designed and constructed to allow for realtime long-term monitoring of brake performance parameters such as the temperature, deceleration, hydraulic line pressure, and vehicle speed. The focus of the design is low cost, small size, and low weight. It will be shown later in the paper that the proposed device has successfully been employed to measure typical brake performance parameters and to examine the driving conditions likely to be traversed during the life of the brake system. Using the proposed design, it is possible to record and analyse vehicle braking parameters over an extended period of time. This design proved superior to existing commercial loggers which are normally too costly and too bulky for efficient in-car testing applications. For the benefit of the reader, the next section presents a background discussion on the brake performance parameters.

\section{BRAKE TESTING PARAMETERS}

The performance of a brake system is assessed by the rate at which this system absorbs the kinetic energy of a vehicle moving under the effects of its inertia and any mechanical energy provided by its prime mover. As pointed out by Fumi [10], the parameters which govern the brake performance can be divided into enabling parameters and resisting parameters.

The enabling parameters are those parameters which control the value of brake torque needed to dissipate the vehicle kinetic energy. These are mainly the effectiveness of the friction material, used on the pads, and the efficiency of the hydraulic system used to push the pads against the brake discs. While the effectiveness of a specific friction material generally deteriorates as the temperature of the brake pad increases, the efficiency of the hydraulic system is judged by the quality (transient characteristics, attained value, and consistency) of the pressure produced in the brake line.

The parameters which resist the action of the brake torque are those parameters which constitute the kinetic energy of the vehicle, namely the vehicle mass and speed at the start of (and during) the braking process. Agudelo and Ferro [11] suggested that either the vehicle acceleration or the distance travelled by the vehicle, as measured from the instant at which the brakes have been applied, can provide an indication as to the performance of the vehicle brakes, although they did make reference to the need to monitor, and in some cases control, testing based on temperature. In agreement with this concept, Runhau [12] presented a device for monitoring the post-brake travelled distance of vehicles. However, Jones [13] seemed to place more emphasis on the temperature of the friction material during the braking process and, as such, presented a device to provide real-time information on the brake pad thermal conditions.

The novel system to monitor brake performance, presented in this paper, not only collects real-time data on both the acceleration of the vehicle and the temperature of the brake pads; it also monitors the performance of the hydraulic system by feeding back real-time information on the pressure in the brake hydraulic lines. The acceleration is measured in two perpendicular directions (along the vehicle centreline and vertically perpendicular to it) to account for the vehicles moving on slopes and hills. Moreover, the proposed system features highly sophisticated electronics and software designs which render the system suitable for remote monitoring applications as needed. The next section offers an insight into the hardware design of the novel brake-monitoring system proposed in this paper. 


\section{HARDWARE DESIGN}

The main aspects of the brake-monitoring system presented here are shown in the block diagram given in Fig. 1. The system has been built upon embedded personal computer (PC) hardware and use of some commercially available peripherals together with hardware developed specifically for the task.

The first requirement of the system was to make measurements of the relevant physical parameters in order to display them. All these parameters are converted to electrical signals by their respective sensors. The logging unit then takes these electrical signals to convert them into a digital form. Subsequently, the software takes over the filtering of data before undertaking tasks to display and store the processed information. The connections to the unit are as follows:

(a) four 0-5 V analogue inputs;

(b) two type- $\mathrm{K}$ thermocouple inputs;

(c) a built-in dual-axis accelerometer (configured for longitudinal and vertical accelerations); (d) two transistor-transistor logic (TTL) inputs, one of these also connected to timer hardware for frequency measurement;

(e) two TTL outputs available for triggering external devices or otherwise;

(f) an external liquid crystal display (LCD) module to display parameters.

A PC/104 form factor was chosen for all the PCrelated hardware, which is standard for small PC hardware to use for portable and industrial-type applications. A motherboard was chosen with a 586 processor running at $166 \mathrm{MHz}$ with $64 \mathrm{MB}$ randomaccess memory and many built-in peripheral adaptors (including keyboard, video graphics array, parallel printer, RS-232, and hard-disk drive) and with a PC/104 bus. Digital-to-analogue conversions and timing and digital input/output ( $\mathrm{I} / \mathrm{O})$ connections are made using a data acquisition (DAQ) card that sits on the PC/104 bus. The DAQ card has eight differential analogue inputs, eight pins of digital input, eight pins of digital output, together with timing and analogue triggering connections. The

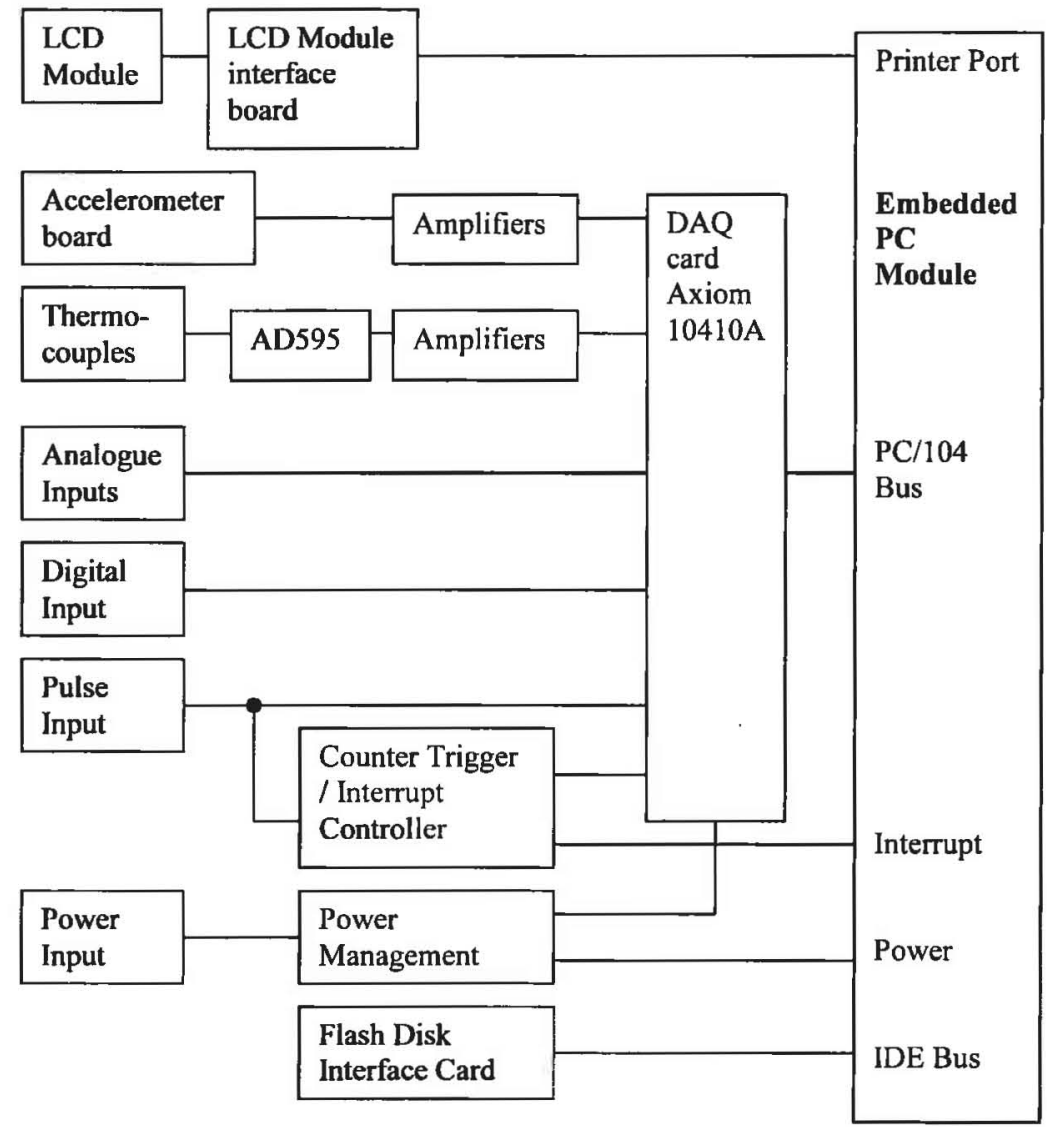

Fig. 1 A block diagram showing the structure of the proposed system 
analogue inputs are set to work over the $0-5 \mathrm{~V}$ range.

For reliability reasons, it was resolved to avoid mechanical hard-disk drives because of their sensitivity to shock and vibration. Compact flash (CF) cards were chosen for mass storage since they have no moving parts. An interface (also of a PC/104 form factor) was sourced that allowed the card to be accessed using an integrated drive electronics (IDE) interface (parallel hard-disk connector). From an operational point of view the CF card relates to the operating system just like a normal hard-disk drive does and so no changes were needed to support its use.

The system runs off the nominal $12 \mathrm{~V}$ power available from a vehicle's electrical network. To convert this into the $5 \mathrm{~V}$ required by the electronics, the final PC/104 module in the system is a switchmode power supply. Keeping with the low cooling requirements, a switch-mode power supply efficiently drops the voltage to the required level, allowing for the input voltage to range between 9 and $18 \mathrm{~V}$.

A 64 by 240 pixel monochrome LCD module was used to display the measured vehicle parameters. The module chosen has a built-in display controller and requires only an 8 bit parallel interface to transfer data.

The four PC/104 modules are arranged in two stacks of two modules, the motherboard and DAQ card on one side (as these cards were required to be coupled via the full $\mathrm{PC} / 104$ bus), and the power supply and CF module on the other side. Both these stacks are mounted onto a baseboard that serves a number of purposes.
The baseboard connects power between the two stacks of $\mathrm{PC} / 104$ modules and also contains other power supply circuitry to generate a bipolar $( \pm) 12 \mathrm{~V}$ supply for use in the system. It contains circuitry to hold power on and switch it off, based on commands from the central processing unit (CPU), a feature used to allow the software to close all files before shutting down the system. A pair of thermocoupleconditioning integrated circuits has also been provided on the board to convert the low-level nonlinear thermocouple signal from the inputs to a $0-5 \mathrm{~V}$ signal readable by the DAQ card. These also look after the cold junction compensation for the thermocouples. The baseboard also serves to connect all the various parts of the system together, which are the front connector board, the accelerometer board, and the PC/ 104 boards. An annotated photograph of the baseboard is shown in Fig. 2.

To be able to measure the frequency of an input signal, the counter input requires an input that is active for 1 cycle of this signal. In addition, the CPU must be notified that a cycle is complete and the counter should be read. A circuit made up of a number of logic integrated circuits generates the appropriate gating and interrupt signals from the pulse input.

The display module connects to the PC using the parallel printer port. This port provides an 8 bit bidirectional data path along with four dedicated output pins and five dedicated input pins. The LCD module requires five control inputs so that some logic is provided to generate these five signals from the four printer port outputs. The data bus ( 8 bits) is connected straight through from the printer port to the LCD. In addition to manipulating the control

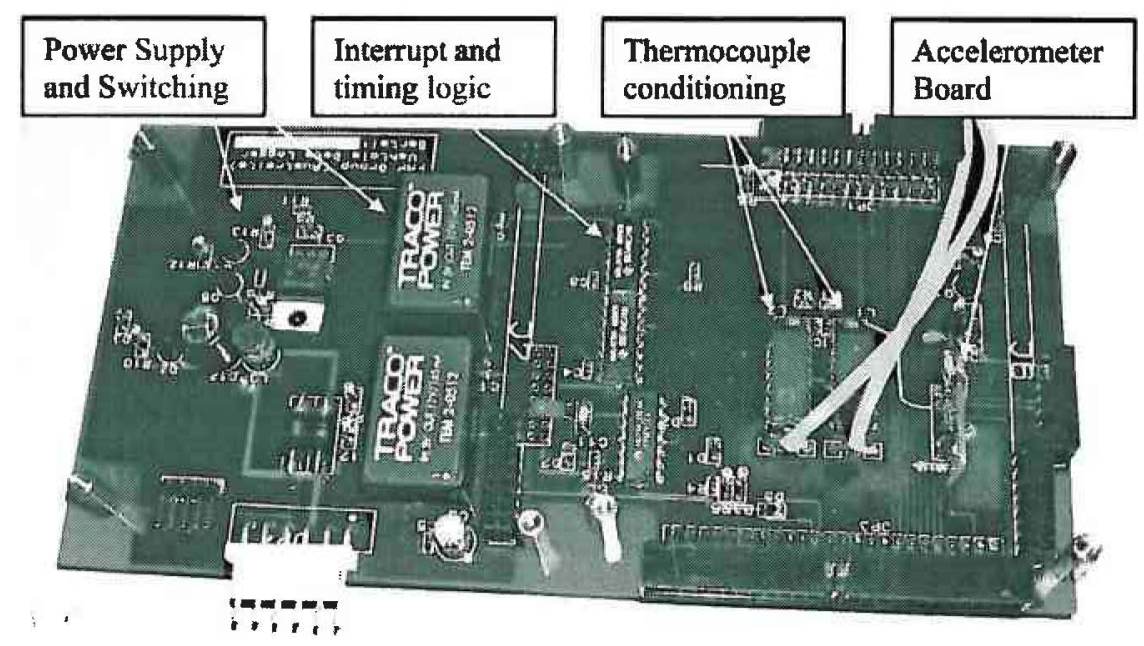

Fig. 2 The baseboard developed for the unit 
signals, power to the module is synchronized with the reset input to ensure that the timing meets the specifications.

The built-in accelerometer is of the microelectromechanical system type, giving sufficient accuracy and bandwidth combined with small size. The output of the sensor is a $0-5 \mathrm{~V}$ signal where the ratio of the signal to the power supply is proportional to the instantaneous acceleration. The sensor provides measurements on two axes. As the unit is intended for brake system measurements where braking is performed in a straight line, the accelerometer was arranged such that one axis nominally aligns to the fore-aft (braking direction) and the other nominally in a vertical direction (up-down). This arrangement allows for angular misalignment of the unit, relative to the vehicle about an axis across the car, to be compensated for mathematically. Figure 3 illustrates the arrangement. The reason for this is to make alignment of the unit simpler while still giving an acceleration reading in the direction of braking. Acceleration in terms of road speed is not as relevant to braking measurements (in terms of brake effectiveness), owing to incline effects. Obviously the road speed is important in terms of energy input but it is not always an accurate indicator of instantaneous brake output.

As shown in Fig. 4, the system consists of two enclosures: the first contains the PC/104 hardware, baseboard, and accelerometer; the second is the display and keypad unit that is placed in the view of the driver. The system can also run without the display unit connected, for logging-only applications. The next section offers an insight into the software package designed to operate the data-logging system.

\section{SOFTWARE DESIGN}

A brief block diagram is shown in Fig. 5 to indicate the structure of the software package which has been written to drive the brake-performance-monitoring system presented in this paper. The software for the

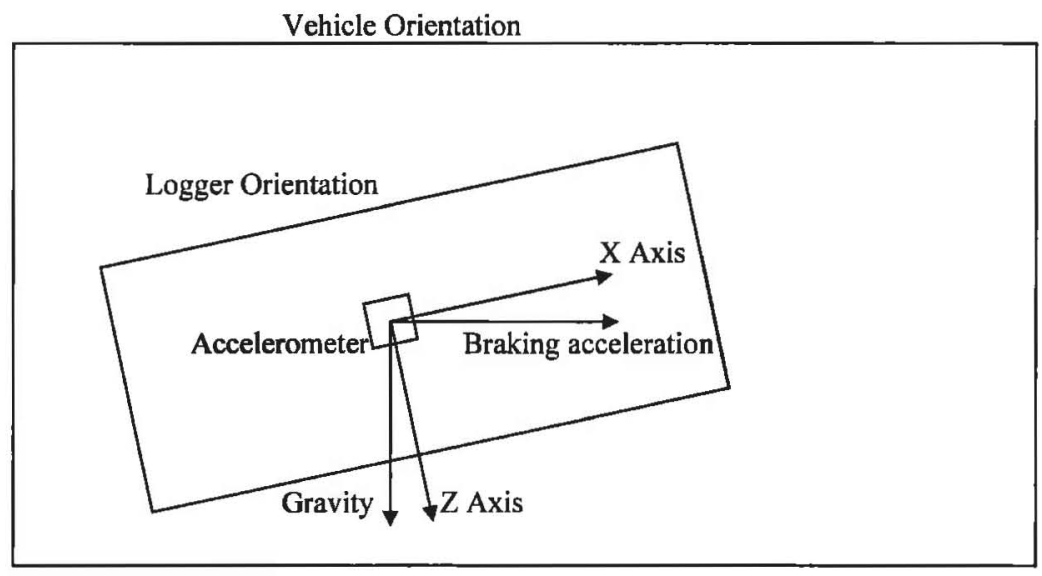

Fig. 3 A diagram of the accelerometer and vehicle orientation

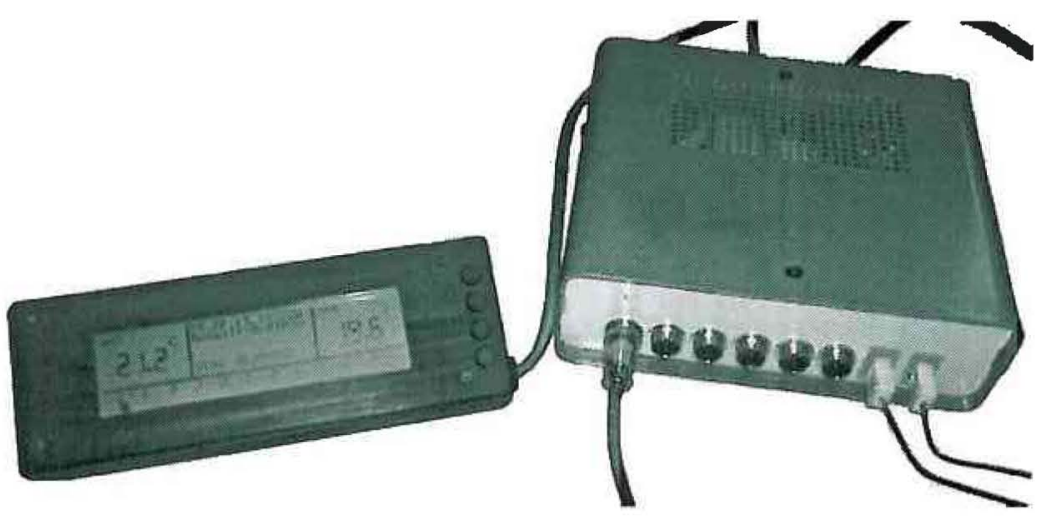

Fig. 4 System hardware enclosure and display 


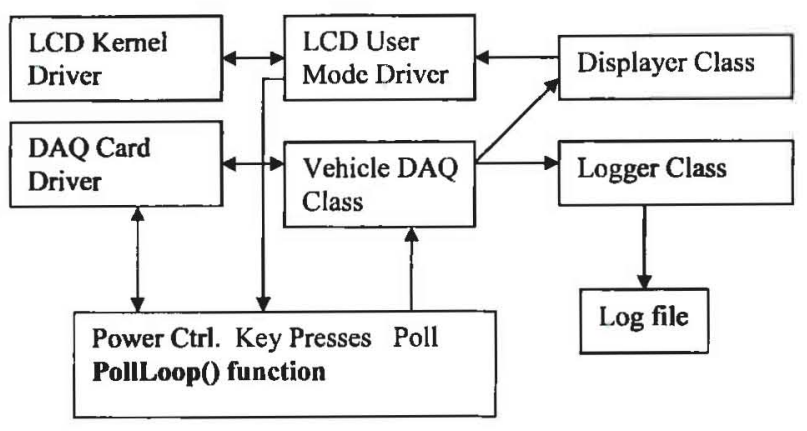

Fig. 5 A block diagram for the control software

unit was written in $\mathrm{C}$ and $\mathrm{C}++$. The unit runs the Linux operating system, specifically a cut-down version of Redhat 6.2 .

A kernel was compiled specifically to suit the unit, to minimize its size, and to reduce the number of kernel modules required to make the system run. This was then built into drivers for the display and DAQ card, data-processing software, a shell, and a number of applications required to maintain the system (e.g. a text editor and some other utility commands). Libraries have also been included to support the installed programs, which occupy less than $10 \mathrm{MB}$ of space on the CF card. The driver for the DAQ card was built as a loadable kernel module so that low level interrupt support, kernel memory, I/O address access, and low level kernel support were available. The driver allows for constant sample rate analogue DAQ (using hardware timing) and frequency-period measurement using a counter. It also manages the $\mathrm{I} / \mathrm{O}$ pins that are used to turn on the power hold function and digital outputs.

Data from the analogue-to-digital converter runs through a first-in first-out buffer to allow the user program to collect data at its own rate, and the interface provided looks to applications much like a file. It is opened and closed like a file (in the same fashion as all Linux drivers) and data are communicated by read and write commands on the file handle. Reading from the file yields data from the analogue information in a binary form to be decoded. For data to be transferred asynchronously (such as reading and setting $\mathrm{I} / \mathrm{O}$ pin values), I/O control calls are used. These are intended for such tasks as controlling serial port baud rates and other out-of-band communication.

Likewise, the display and keypad also use a kernel driver module to form the software interface between the user program and the hardware. This provides display functionality, allowing data to be sent to the display. It also provides access to the keypad.
The user mode software performs three basic functions. It takes the data from the analogue inputs and performs calibration and filtering to yield data ready to be stored. The calibration is performed purely in software to avoid the need for physical adjustment, and each input is assigned a name and a physical unit reference so that, whether it is displayed or recorded to flash drive, it is still traceable.

The software can then record the measured and conditioned data to the flash drive. In order to minimize the amount of recorded data (while still making use of easily post-processed text data), the system is able to adjust the sample rate depending on the inputs from the various sensors. This was found to be particularly effective with long-term brake performance monitoring, where the system recorded at $10 \mathrm{~Hz}$ during brake applications (line pressure above $0.4 \mathrm{MPa}$ and speed above $3 \mathrm{~km} / \mathrm{h}$ ) and $0.1 \mathrm{~Hz}$ off-brake (pressure less than $0.4 \mathrm{MPa}$ or speed less than $3 \mathrm{~km} / \mathrm{h}$ ). Logging can be disabled globally or only some channels can be recorded, although all channels are recorded at the same rate.

The software also updates the LCD with data as configured by the user. Various channels can be sent optionally to different parts of the screen including a moving bar at the bottom. The moving bar is useful to assist a brake test driver in achieving and maintaining a particular deceleration or line pressure. Following a moving bar is significantly less demanding of concentration for a driver than interpreting changing numbers. The bar update rate is adjustable but was set to $20 \mathrm{~Hz}$ on the prototype, which proved easy to see, whereas the numerical displays are easily readable only up to $2 \mathrm{~Hz}$.

The software also performs some 'housekeeping' tasks such as monitoring keypad presses, current power state (if the vehicle ignition is on), and also mounting and unmounting the logging file system to ensure the integrity of recorded data. The procedure used to set up and calibrate the proposed system is briefly outlined in the next section.

\section{SETTING AND CALIBRATION}

Settings for the units are contained in a text file. There are per-channel settings for calibration, identification, and filtering (i.e. smoothing). The logging module has been equipped with settings to control the channel list and logging rate. The display module has been provided with options to configure what is shown on the screen. Analogue input channels require calibration in the forms of an offset 
and a scale factor. Only linear sensors' inputs are accounted for in the current version of the software.

The $X$ and $Y$ accelerometer channels are processed to yield an acceleration signal by determining the component of acceleration in the direction of motion upon braking. The angle is found by setting the vehicle onto a level surface to measure the acceleration signals while stationary. Calibration of the $X$ and $Y$ accelerometer channels is performed by aligning the unit for maximum (or minimum) reading from the respective axis. The input thus obtained represents a positive or negative $1 g$ acceleration.

The speed signal and the distance measurements have only a scale factor; no offset calibration is provided as it is not required. Calibration of the speed and odometer channels requires knowledge of the distance between consecutive input pulses or the number of pulses per unit of distance. The calibration factor is simply the distance per pulse in the appropriate units; kilometres have been used in the present research project.

Calibration of other inputs (e.g. pressure, temperature, and force) can be made using one of two methods: either the sensor can be connected to the unit and a predefined input is applied, or a transducer simulator is used in the case of sensors which are difficult to expose to a calibrated reference (such as thermocouples). The system software is also equipped with the capability to effect settings on a per-channel basis, such as the channel name, engineering units, damping, and hysteresis parameters.

The next section offers details on the outcome of experimental effort conducted using the system proposed in this paper.

\section{DEVICE IMPLEMENTATION}

To simplify installation and set-up of the logger, the only data recorded during the validation testing were the deceleration, speed, distance travelled, line pressure, and brake rotor temperature (using a non-contact infrared pyrometer). Each data record has a time stamp allowing the time of the sample to be resolved to the nearest $10 \mathrm{~ms}$ (using a $100 \mathrm{~Hz}$ sampling rate). The logger has the ability to record at two different sample rates. This feature was provided to allow for one sample rate to cover driving conditions where much detail is required (higher rate), and a second to account for when less information is required. Up to two input channels can be monitored and only when each satisfies the criteria will the logger move to the higher sample rate.
With the focus on the brake systems, the data of greatest importance are the in-stop data. Given this focus, the two criteria chosen to determine whether the brakes were being applied at a given instant were, first, whether there was brake line pressure (brake pedal pressed down) and, second, whether the speed was above a small nominal value (vehicle moving). The pressure was chosen to be $0.4 \mathrm{MPa}$ in the testing presented here and the vehicle's speed was above $3 \mathrm{~km} / \mathrm{h}$. This means that, for the purpose of the analysis, a stop was considered to consist of a single depression of the brake pedal with the car initially moving, whether or not it ended with the car stationary. The process of gathering data during the test was as simple as downloading the contents of the memory card from the logger as it became full. Because the driving patterns change from drive to drive and because the logging rate changes dynamically upon brake application, the amount of data logged each day varied.

The vehicle used for testing was a Holden Commodore VX, fitted with a prototype VE (2006) brake system. Testing loops of about $400 \mathrm{~km}$ each were selected to cover a variety of open highway driving, medium-speed urban driving, and low-speed inner-city driving patterns. The vehicle travelled a distance of about $20000 \mathrm{~km}$.

A number of connections were made between the unit, the vehicle and the various sensors to provide the necessary functionality.

For a power supply, a connection to an always-on (unswitched) fused battery power was made. A second connection to the accessory circuit (switched by the key) provides an activate signal. When the accessory circuit is powered up, the logger boots and, when the key is turned off, the unit takes power from the unswitched circuit until the system can be safely powered down. During shutdown the data Iog file is closed and the file system unmounted.

A connection was tapped into the wire between the speed sensor and the engine control computer. This signal is a square-wave $12 \mathrm{~V}$ peak-to-peak type, which corresponds to a movement of the vehicle of approximately $0.3 \mathrm{~m}$. The speed and distance signals have been calibrated in comparison with the vehicle's speedometer and odometer. These have proven to be adequately accurate for the purpose of this study.

A pressure sensor has been plumbed into the front-brake hydraulic system to allow the line pressure to be recorded. Hence, a multi-core wire was run between the sensor and the logger (fitted under the passenger seat) to carry both power to the 
sensor and the pressure signal back to the logger. The pressure sensor is capable of measuring up to $10 \mathrm{MPa}$ with a burst pressure of greater than $20 \mathrm{MPa}$. It is unlikely even during emergency stops that the brake line pressure would exceed $10 \mathrm{MPa}$.

An LCD unit is connected to the data logger to help to verify the correct operation of the system, and to allow the driver to verify that the unit has powered down correctly. The display should not influence the driving style and safety of the drivers, and for this reason the display was placed in a pocket at the rear of the front seat where it could be viewed easily while stopped but was out of view while driving.

\section{EXPERIMENTAL RESULTS}

The proposed device was successfully and reliably used to conduct a myriad of experimental runs that spanned a total travelled distance of $20000 \mathrm{~km}$. The results of these runs are intended to reflect on the brake performance, and to guide future brake design endeavours. The outcome of a single-loop driving test is shown by way of example in Fig. 6 . The figure reveals a number of features that can be seen in the stop. For example, the application of brake pressure takes place within the first second of the stop. After the initial brake application the pressure is released somewhat to what appears to be the driver's intended deceleration rate. The undulations in the acceleration level are probably the driver's response to external factors such as other vehicles and adjustments in the deceleration level, resulting in the vehicle stopping at the correct point.

Figure 7 shows the brake effectiveness, speed, and temperature with respect to time. The brake effectiveness is the ratio of deceleration to pressure. In its usual form it is a measure of the friction coefficient of the brake interface, which is dimensionless and calculated from the individual brake torque (e.g. on a dynamometer). In the case of in-vehicle testing where the contribution of each wheel to the total retardation force is not known, it is sufficient to express the effectiveness in such units as the acceleration per unit pressure, and that is what is plotted in the figure. There does not appear to be a strong relationship of the effectiveness to either the speed or the temperature in this case.

Figure 8 illustrates the physics of a stop. Looking at the acceleration trace it appears that the driver was attempting to maintain an approximately constant deceleration level, while adjusting the level to allow for external factors such as nearby vehicles and the desired stopping point. A relatively linear drop in speed can be observed; the integrating effect of the speed measurement relative to acceleration gives a much smoother velocity curve relative to decelera-

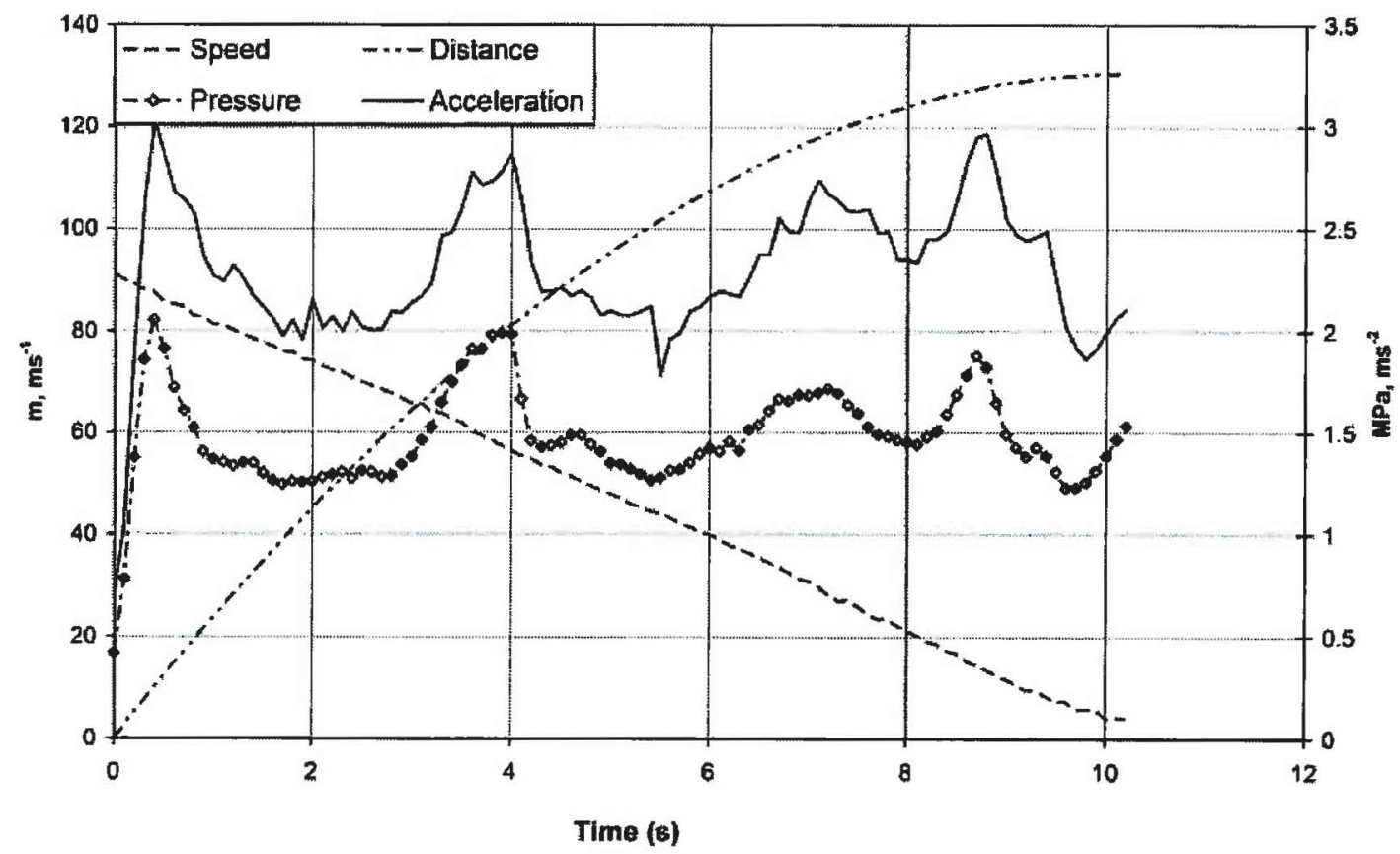

Fig. 6 Various pararneters measured with the brake-monitoring system 


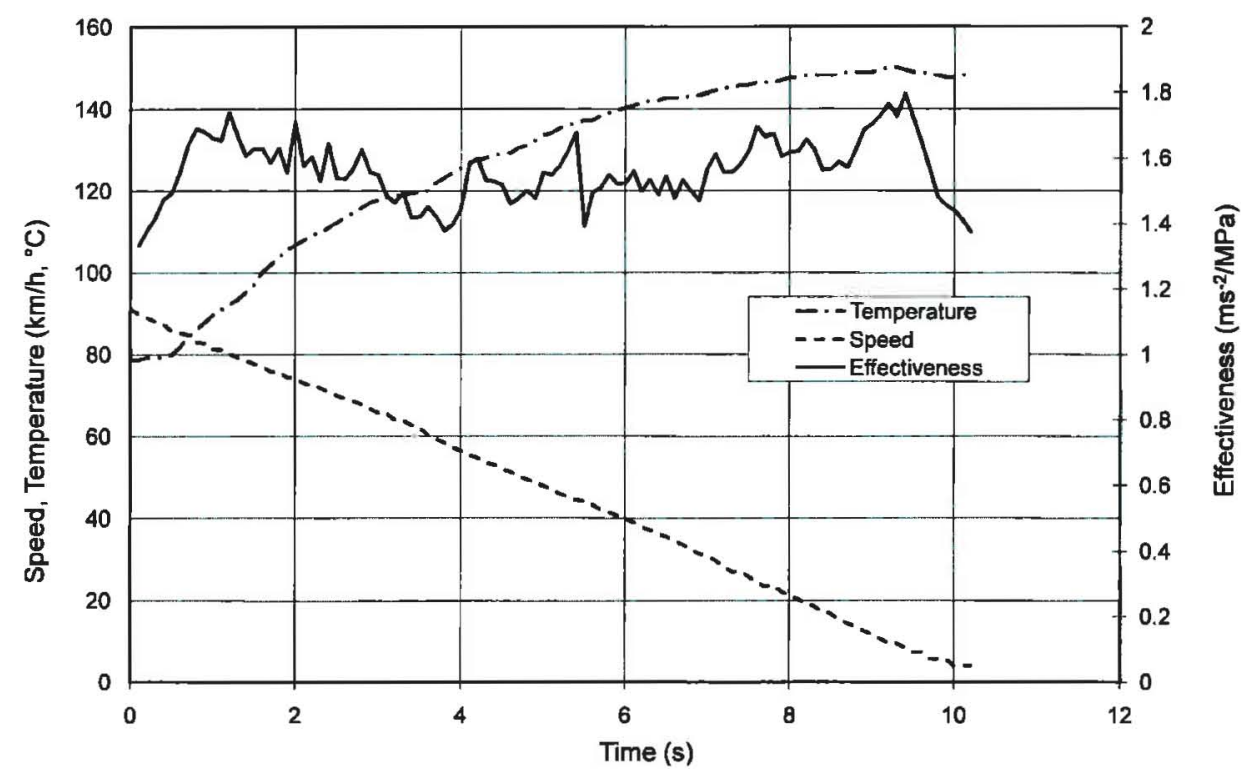

Fig. 7 A representation of the brake effectiveness

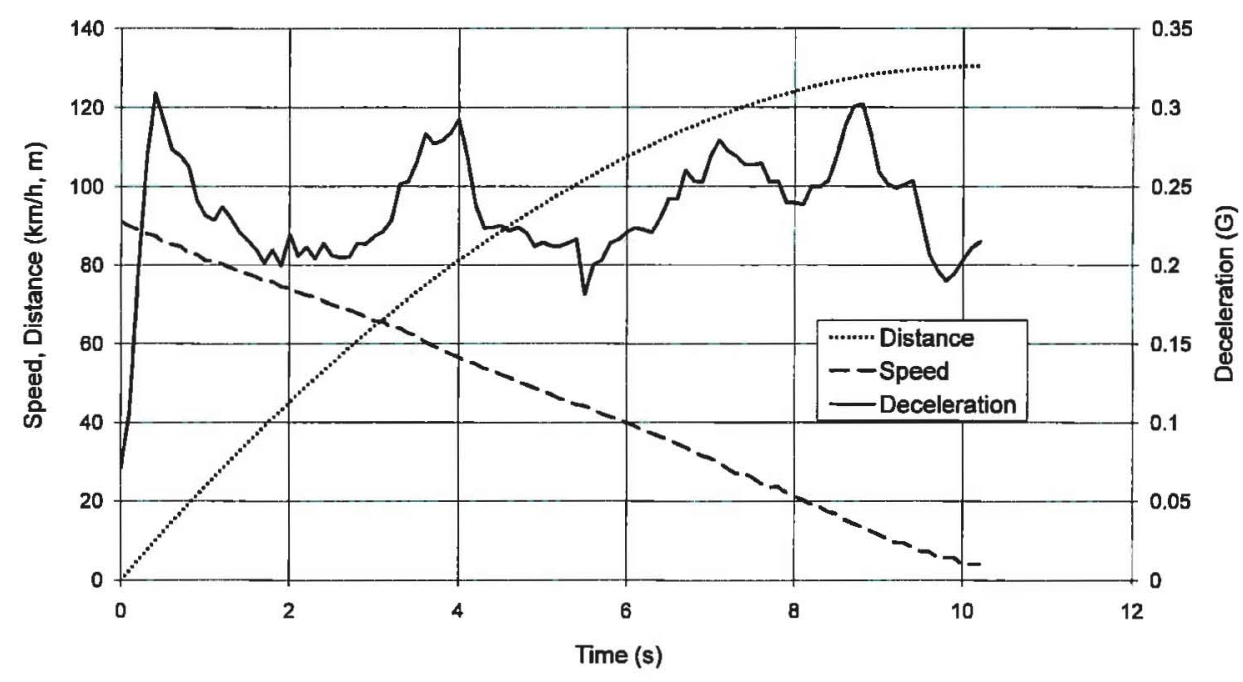

Fig. 8 Mechanical parameters as revealed by the system

tion. The time-displacement curve shows an approximate parabolic function as expected for a nearlinear speed versus time curve. The graphs show the expected relationship between these vehicle dynamic quantities.

Figure 9 presents a plot of brake line pressure and acceleration, showing both the timing and the magnitude relationships. It can be seen that the acceleration follows the pressure very closely. The relationship between the two is dependent on a number of factors such as the size and friction level of the brake pads, the hydraulic properties of the brake calliper, and the amount of engine braking. It is interesting to note that the relationship between the brake pressure and the deceleration is not dependent on the slope of the road. If the car is going down a hill with no braking force or power input from the engine, then the acceleration measured by the brake logger will be zero, even though the speed of the car is increasing. Conversely, travelling up a hill at a constant speed (but with some accelerator pedal depression to maintain that speed), the logger will be measuring positive acceleration despite the fact that the vehicle's speed remains constant.

Further analysis of the data from a whole loop shows other information relevant to brake design in general. In these plots the data from a whole loop 


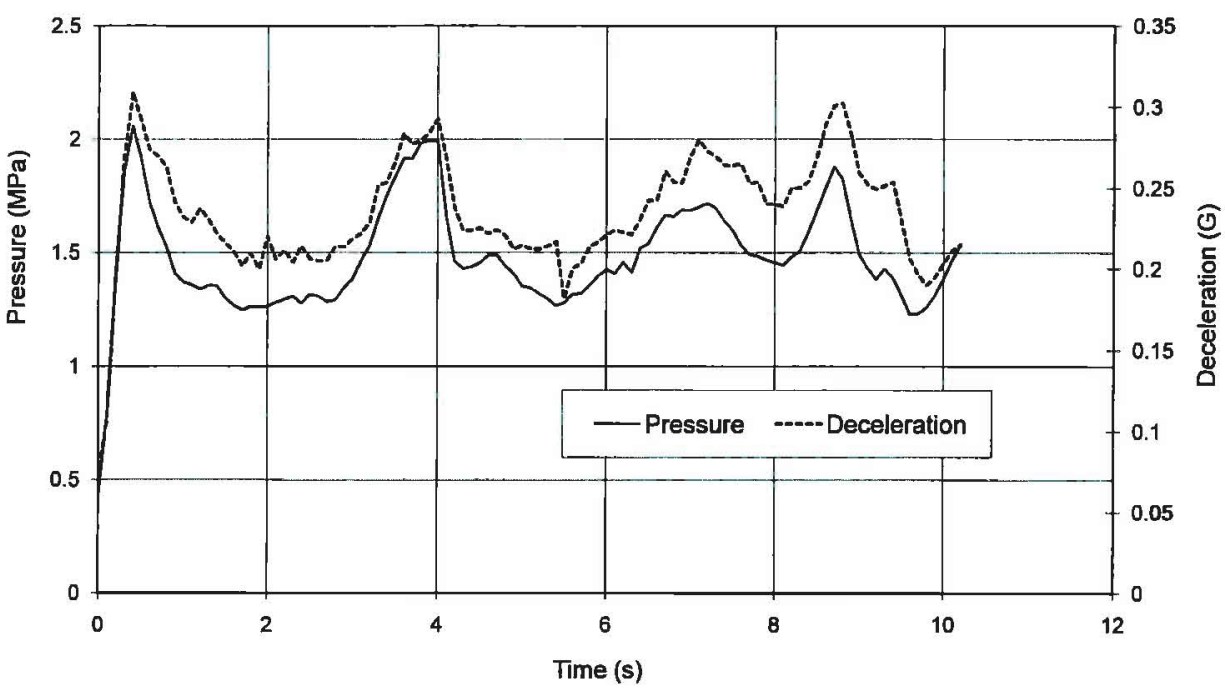

Fig. 9 Pressure and temperature signatures

were separated into braking events and off-brake events. The plots shown illustrate for a daytime drive the distribution of a few of the parameters measured. The cycle yielded approximately 450 stops.

Figure 10 shows a histogram of the time-averaged in-stop deceleration levels for the drive. The emphasis on lower deceleration levels is notable, with the majority of stops at less than $0.15 \mathrm{~g}$. This is opposed to current brake performance and wear dynamometer test procedures, which cover deceleration levels up to significantly higher levels, sometimes up to $\mathrm{lg}$.

Figure 11 presents a histogram of time-averaged line pressure levels. Given that the deceleration is a function of the friction level and the pressure, it is logical that the shape of this distribution is similar to that of the deceleration.

Figure 12 gives a histogram of the end speed of each stop. Stops of less than $1 \mathrm{~s}$ and stops beginning at a speed of less than $15 \mathrm{~km} / \mathrm{h}$ were excluded. This chart shows that, although the $0-10 \mathrm{~km} / \mathrm{h}$ bin has the largest number of stops of all bins, overall the majority of brake applications do not bring the vehicle to a complete stop.

\section{CONCLUSIONS}

The most highlighted aspects of a road vehicle are those which relate to the safety of the drivers and

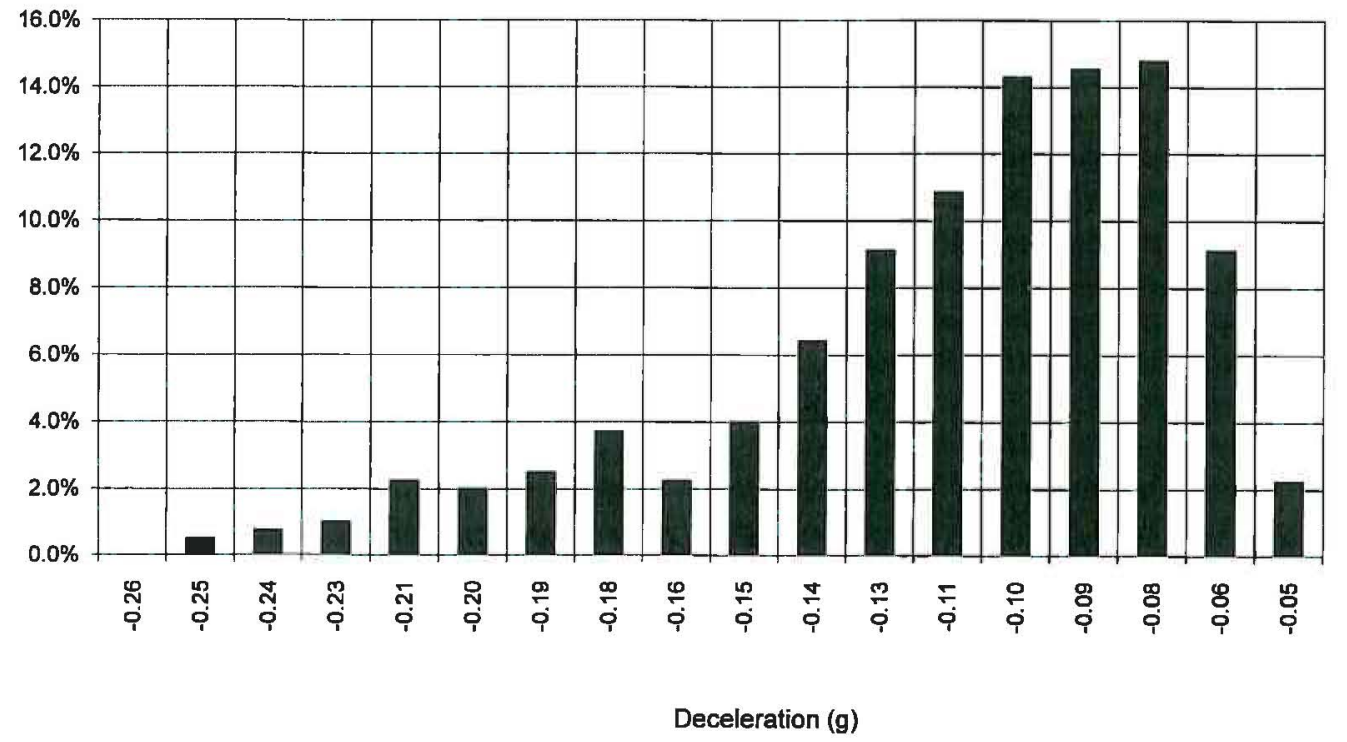

Fig. 10 A histogram of average in-stop deceleration 


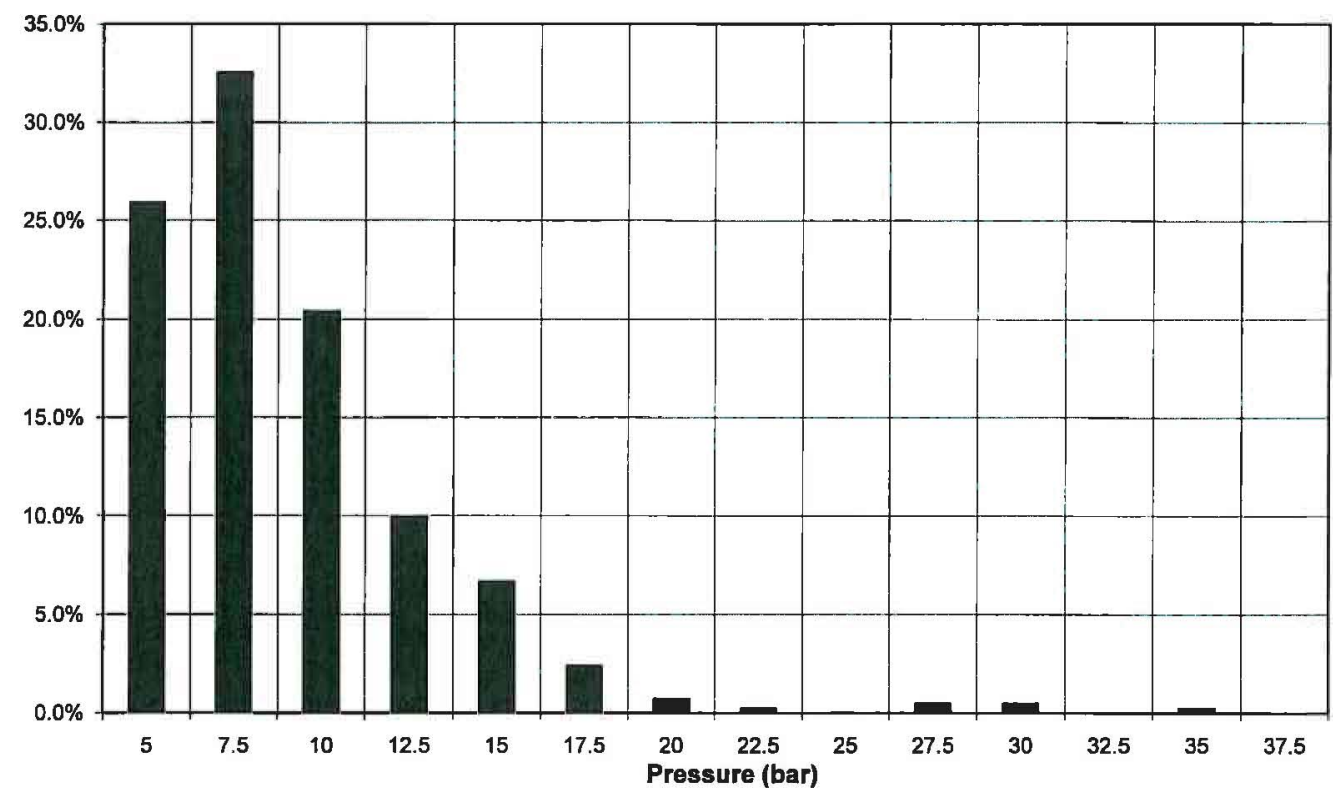

Fig. 11 A histogram of average in-stop line pressure

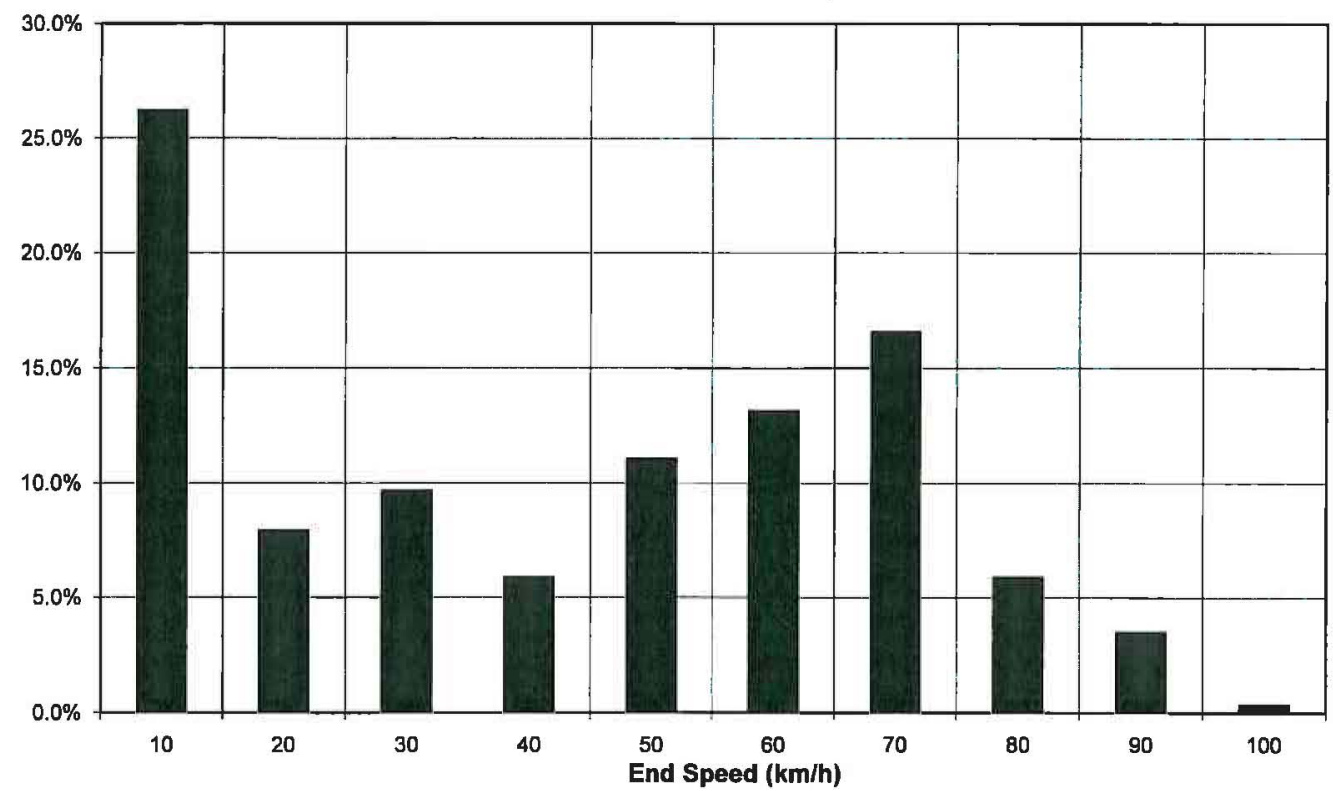

Fig. 12 A histogram of brake application end speed

passengers. Monitoring the performance of a brake system makes it possible to conduct real-time assessment of its various components and to decide on whether the design is adequate to achieve the desired performance outcomes. The work presented in this paper features research effort undertaken to design, construct, and test a novel in-car brakemonitoring system. The various measurable parameters relevant to brake performance have been discussed and a fundamental subset of them was then selected for real-time monitoring and reporting.

The novel in-car brake-monitoring system proposed in the paper has been successfully employed in an extensive procedure, which featured running of a test vehicle over a network of long and short routes for a period of time. The collected data were then analysed to confirm the validity of the proposed 
system, and the analysis of the collected data revealed various interesting aspects of brake systems.

\section{REFERENCES}

1 Chand, N., Hashmi, S. A. R., Lomash, S., and Naik, A. Development of asbestos free brake pad. Instn Engrs (India) J., Mech. Engng, 2004, 85(4), 13-16.

2 Rienstra, S. A. and Rietveld, P. Speed behaviour of car drivers: a statistical analysis of acceptance of changes in speed policies in The Netherlands, Series Research Memoranda 0007, Faculty of Economics, Business Administration and Econometrics, VU University Amsterdam, The Netherlands, 1996, available from ftp://zappa.ubvu.vu.nl/19960007.pdf.

3 Shiiba, T. and Suda, Y. Evaluation of driver's behavior with multibody-based driving simulator. Multibody System Dynamics, 2007, 17(4), 195-208.

4 Igarashi, K., Takeda, K., Itakura, F., and Abut, H. Is our driving behaviour unique? In DSP for invehicle and mobile systems (Eds $\mathrm{H}$. Abut, J. H. L. Hansen, and K. Takeda), 2005, pp. 257-274 (Springer-Verlag, New York).

5 Erdogan, H., Ercil, A., Ekenel, H. K., Bilgin, S. Y., Eden, I., Kirisci, M., and Abut, H. Multi-modal person recognition for vehicular applications. In Proceedings of the Sixth International Workshop on Multiple classifier systems (MCS 2005), Lecture Notes in Computer Science, vol. 3541, Seaside,
California, USA, 13-15 June 2005, pp. 366-375 (Springer-Verlag, Berlin).

6 Smith, P., Shah, M., and Da Vitoria Lobo, N. Determining driver visual attention with one camera. IEEE Trans. Intell. Transpn Systems, 2003, 4(4), 205-218.

7 Sakamoto, H. Heat convection and design of brake discs. Proc. IMechE, Part F: J. Rail and Rapid Transit, 2004, 218(3), 203-212. DOI: 10.1243/ 0954409042389436.

8 Park, J.-P. and Choi, Y.-S. Brake squeal noise due to run-out. Proc. IMechE, Part D: J. Automobile Engineering, 2007, 221(7), 811-821. DOI: 10.1243/ $09544070 J A U T O 342$.

9 Fieldhouse, J. D., Steel, W. P., and Talbot, C. J. The measurement of the absolute displacement of a noisy disc brake. Proc. IMechE, Part D: J. Automobile Engineering, 2008, 222(7), 1121-1140. DOI: 10.1243/09544070JAUTO582.

10 Fumi, D. E. An in-vehicle data acquisition system to monitor the service conditions of brake systems. Master's Engineering Science Thesis, University of Ballarat, Victoria, Australia, 2008.

11 Agudelo, C. and Ferro, E. Technical overview of brake performance testing for original equipment and aftermarket industries in the US and European markets. Technical report FEV205-01, Link Testing Laboratories Inc., 2005.

12 Runhau, G. Brake performance monitoring. Int. Pat. WO 2006/018089 Al, 2006.

13 Jones, L. B. Method and apparatus for thermal testing of brake performance. Int. Pat. WO 99/04236, 1999. 